\title{
Advances in the Study of the Potential Hepatotoxic Components and Mechanism of Polygonum multiflorum
}

\author{
He-Shui Yu, ${ }^{1,2}$ Lin-Lin Wang, ${ }^{3}$ Ying He, ${ }^{3}$ Li-Feng Han $\left(\mathbb{D},{ }^{2}\right.$ Hui Ding, ${ }^{1}$ Xin-Bo Song, ${ }^{1,4}$ \\ Xiu-Mei Gao (D), ${ }^{2}$ Nai-Ru Yun ${ }^{(D)},{ }^{3}$ and Zheng Li ${ }^{1,2}$ \\ ${ }^{1}$ College of Pharmaceutical Engineering of Traditional Chinese Medicine, Tianjin University of Traditional Chinese Medicine, \\ Tianjin 301617, China \\ ${ }^{2}$ Tianjin State Key Laboratory of Modern Chinese Medicine, Tianjin University of Traditional Chinese Medicine, \\ Tianjin 301617, China \\ ${ }^{3}$ Second Affiliated Hospital of Tianjin University of Traditional Chinese Medicine, Tianjin 300250, China \\ ${ }^{4}$ Tianjin Modern Innovative TCM Technology Co., Ltd., Tianjin 300000, China
}

Correspondence should be addressed to Nai-Ru Yun; yunnairu987@126.com and Zheng Li; lizheng@tjutcm.edu.cn

Received 2 June 2020; Revised 26 August 2020; Accepted 14 September 2020; Published 30 September 2020

Academic Editor: Jie Liu

Copyright (c) $2020 \mathrm{He}$-Shui Yu et al. This is an open access article distributed under the Creative Commons Attribution License, which permits unrestricted use, distribution, and reproduction in any medium, provided the original work is properly cited.

The roots of Polygonum multiflorum (PM) (He Shou Wu in Chinese) are one of the most commonly used tonic traditional Chinese medicines (TCMs) in China. PM is traditionally valued for its antiaging, liver- and kidney-tonifying, and hair-blackening effects. However, an increasing number of hepatotoxicity cases induced by PM attract the attention of scholars worldwide. Thus far, the potential liver injury compounds and the mechanism are still uncertain. The aim of this review is to provide comprehensive information on the potential hepatotoxic components and mechanism of PM based on the scientific literature. Moreover, perspectives for future investigations of hepatotoxic components are discussed. This study will build a new foundation for further study on the hepatotoxic components and mechanism of PM.

\section{Introduction}

The occurrence of adverse reactions caused by TCMs and their agents has increased, as they are widely used as disease treatments, in healthcare, etc. The liver, as an important drug-metabolizing organ, is particularly vulnerable to damage. PM, as a traditional tonic medicine, has antiaging, liver- and kidney-tonifying, hair-blackening, etc. effects [1-3].

However, PM-induced hepatotoxicity cases have been frequently reported in China and other countries. Hepatotoxicity cases caused by PM were reported by the Medicines and Healthcare Products Regulatory Agency (MHRA) [4-6]. Then, the China National Medical Products Administration (NMPA) also issued a notice on strengthening the supervision of health foods containing PM. Additionally, "Focus on Risk of Liver Damage Caused by Oral PM and Its Preparation" was published in "Adverse Drug Reaction Information Bulletin" by National Center for ADR
Monitoring, China. As PM and its preparations are widely used in the treatment and prevention of diseases, the hepatotoxic effects of PM-based products limit its long-term usage, and the safety of PM requires attention.

The clinical hepatotoxic feature of PM was summarized according to the literature. The ingestion of PM formulations or preparations leads to liver injury, including PM soaked in wine after steaming, PM dried and ground into powder after steaming, oral liquid containing PM, capsules containing PM, Shou Wu tea, etc. PM and its preparations have been reported to lead to liver injury after injection for several days to several months and at a large range of doses. The ingestion of PM leads to liver injury in many populations, including the general population and people with hair loss, white hair, vitiligo, itchy skin, high blood pressure, coronary heart disease, cerebrovascular stenosis, high cholesterol, dermatitis, eczema, acne, and cerebral infarction. The clinical features of liver injury that result from the ingestion of PM or its preparations include nausea, 
vomiting, diarrhea, abdominal pain, nervous, restlessness, difficulty breathing, tic, upper gastrointestinal bleeding, hybrid hepatitis, cholestasis hepatitis, etc. The ingestion of PM leads to liver injury in people aged $5 \sim 78$ years, and the occurrences in men and women are almost equal. In conclusion, the clinical features of liver injury that result from the ingestion of PM are not definitively related to processing, duration, dosage, age, gender, complications, etc. [4-10] (Table 1).

However, although PM extracts are generally considered to be relatively safe, PM extracts may still show hepatotoxicity. Most ancient books recorded that PM is not poisonous, but a small number of ancient books, such as "Ben Cao Hui Yan," recorded that raw PM is qi cold and cold having property of contraction, and raw PM is poisonous. PPM is qi warm and not poisonous. These prove that PM contains compounds that could induce adverse reactions. Unfortunately, although extensive experiments have been performed both in vivo and in vitro in recent years, the potential toxic components and possible mechanisms that cause liver injury remain unclear.

The aim of this review is to provide comprehensive information on the potential hepatotoxic components and mechanism of PM based on the scientific literature. Moreover, perspectives for future investigations of hepatotoxic components are discussed. This study will build a new foundation for further study on the hepatotoxic components and mechanism of PM.

\section{Study of Potential Hepatotoxic Compounds in PM}

Modern research on PM begins with the study of its composition. Stilbenes, anthraquinones, procyanidins, flavonoids, phospholipids, catechins, and other compounds were isolated and identified from PM [15-17]. All these compounds play indispensable roles in the pharmacological effects of PM. However, scholars have begun to study potential hepatotoxic compounds, as frequent reports of liver injury involve PM. Anthraquinones (AQs), stilbenes, and catechins or their derivatives are the main controversial potentially toxic ingredients.

2.1. AQs or Their Derivatives and Liver Injury. AQs are one of the major potential hepatotoxic compounds in PM. The effects of raw PM and processed PM (PPM) with 70\% ethanol extract on the liver were studied. These results showed that the $95 \%$ ethanol elution of the ethanol extract could inhibit the growth of L02 cells [18-20]. The 95\% ethanol elution of raw PM and PPM ethanol extract are considered to be potential hepatotoxic parts of PM. In addition, researchers found that the order of toxicity was raw PM ethanol extract $>$ raw $\mathrm{PM}$ water extract $>$ PPM ethanol extract $>$ PPM water extract [21-25]. It is worth noting that the content of AQs, such as emodin, and its derivatives were higher or main compounds in PM ethanol extract and 95\% ethanol elution of PM ethanol extract [15], which reveals that AQs may be toxic ingredients. Therefore, the effect of main anthraquinone compounds, such as emodin, rhein, physcion, chrysophanol, etc., on the liver was studied in vivo and in vitro [26-38].

Studies have shown that emodin and rhein can deplete GSH, promote the production of intracellular ROS and the depolarization of the mitochondrial membrane, and upregulate the levels of cleaved Caspase-8, Caspase-9, Caspase-3, etc., inducing the apoptosis in L02 and HepG2 cells [39-43]. AQs and anthranone from PM, such as emodin and chrysophanol, significantly inhibited the activity of the bile salt export pump (Bsep), multidrug resistance-associated protein 2 (Mrp2), and basolateral efflux transporters; downregulated the activity of $\mathrm{Na}+$ /taurocholate cotransporting polypeptide (Ntcp) [44]; altered bile acid (BA) disposition; and resulted in liver injury. While anthraquinone and dianthrone exhibited the strongest inhibitory effect on UGT1A1 activity [34, 45, 46], UGT1A1 is an important UGT isoform involved in the metabolic clearance of bilirubin, which is a toxic waste product of heme degradation [47]. The inhibition of UGT1A1 may lead to bilirubin accumulation, which could induce jaundice, liver injury, etc. [48-50]. In addition, research has shown that the oral administration of emodin to rats can directly induce liver damage, and emodin can induce intracellular oxidative stress and ER stress through the AhR-CYP1A1 pathway and then induce the apoptosis pathways in hepatocytes $[32,33,51]$.

The above results suggest that emodin, rhein, physcion, chrysophanol, emodin-8-O-glc, emodin-O-(acetyl)-hex, emodin-O-hex-sulfate, emodin-O-glc, emodin-O(malonyl)-hex, and other anthraquinone components or their derivatives might be potential hepatotoxic compounds in PM.

However, some studies have noted that the speculation that AQs are the hepatotoxic components in PM lacks scientific evidence. Emodin, as well as other components, has a low bioavailability and might lead to liver damage only at high concentrations. According to the literature, to have liver damage, healthy adults need at least $339 \mathrm{~g}$ of PM in a single oral dose. However, the clinical dose of PM is $3 \sim 6 \mathrm{~g}$ of raw Shou $\mathrm{Wu}$ and $6 \sim 12 \mathrm{~g}$ of PPM $[52,53]$. Thus, the hepatotoxic dose of AQs in PM is far from the actual dose, and the speculation that AQs are the hepatotoxic component of PM needs to be further explored.

2.2. Tannin and Liver Injury. Tannins can cause liver damage in grazing animals and were the main cause of cryptogenic liver damage in the past. The content of tannins in PM is high, up to $15 \%$, and its content decreases as processing time increases [54]. Scholars have studied the role of tannins in liver damage caused by PM and found that tannins could significantly increase serum ALT, AST, AKP, TP, AIB, and TBA enzyme activity and induce significant damage in hepatocytes [55]. The administration of long-term highdoses and short-term medium doses is harmful to the liver in mice, and small doses induce no obvious liver damage. However, liver damage can be restored after stopping drug administration [56]. Research has also shown that gallic acid 
TABLE 1: Retrospective analysis on cases of liver injury of Polygonum multiflorum.

\begin{tabular}{|c|c|c|c|c|c|c|c|}
\hline Case & Age & $\begin{array}{l}\text { Duration of } \\
\text { intake, day }\end{array}$ & Preparations & $\begin{array}{l}\text { Presenting } \\
\text { PM }\end{array}$ & Extrahepatic manifestation & $\begin{array}{c}\text { Type of liver } \\
\text { injury }\end{array}$ & Reference \\
\hline 264 & $5 \sim 63$ & $7 \sim 365$ & $\begin{array}{l}\text { Shou Wu tablet, Yangxue } \\
\text { Shengfa capsule, Yanshou } \\
\text { tablet, Jingwu capsule, Yishen } \\
\text { Wufa oral liquid, Qibao } \\
\text { Meiran pill, Tianma Shouwu } \\
\text { tablet, Huo Li Su oral liquid, } \\
\text { Geng Nian An tablet, Kunbao } \\
\text { pill, Xinyuan capsule, Zhi } \\
\text { Shou Wu granule, Renshen } \\
\text { Shouwu capsule, Fu Fang } \\
\text { Shou Wu oral liquid, Shou Wu } \\
\text { pill, Anshen Bunao liquid, } \\
\text { Jiang Zhi Ling tablet, Bantu } \\
\text { pill, Bushen Yishou capsule, } \\
\text { Huichun Ruyi capsule, } \\
\text { Shenbao tablet, Jiangzhi } \\
\text { Huazhuo capsule, Heishou } \\
\text { Shengfa granule, Baishi pill, } \\
\text { Shou Wu tea, etc. }\end{array}$ & PPM & $\begin{array}{l}\text { Fever, fatigue, loss of appetite, } \\
\text { jaundice, erythra, myalgia, } \\
\text { nausea and vomiting, etc. }\end{array}$ & $\begin{array}{l}\text { Mixed, } \\
\text { hepatocellular, } \\
\text { cholestasis }\end{array}$ & {$[10,11]$} \\
\hline 214 & $19 \sim 78$ & $3 \sim 120$ & $\begin{array}{c}\text { He Shou Wu powder, He Shou } \\
\text { Wu tea, He Shou Wu wine, } \\
\text { etc. }\end{array}$ & Raw PM & $\begin{array}{l}\text { Fever, fatigue, loss of appetite, } \\
\text { jaundice, erythra, myalgia, } \\
\text { nausea and vomiting, ascites, } \\
\text { liver-palms, gray stool, etc. }\end{array}$ & $\begin{array}{l}\text { Mixed, } \\
\text { hepatocellular, } \\
\text { cholestasis }\end{array}$ & {$[9,12]$} \\
\hline 5 & $36 \sim 60$ & $30 \sim 90$ & Runzao Zhiyang capsule & $\begin{array}{c}\text { raw PM and } \\
\text { PPM }\end{array}$ & $\begin{array}{l}\text { Fever, fatigue, loss of appetite, } \\
\text { jaundice, erythra, myalgia, } \\
\text { nausea and vomiting, ascites, } \\
\text { liver-palms, gray stool, etc. }\end{array}$ & $\begin{array}{l}\text { Mixed, } \\
\text { hepatocellular, } \\
\text { cholestasis }\end{array}$ & {$[10,13]$} \\
\hline 326 & $19 \sim 70$ & $5 \sim 180$ & $\begin{array}{c}\text { Huangjing Zanyu capsule, } \\
\text { Huichun Ruyi capsule, } \\
\text { Shenjiao capsule, He Shou Wu } \\
\text { powder, He Shou Wu tea, He } \\
\text { Shou Wu wine, decoction } \\
\text { containing PM, herbal paste, } \\
\text { healthcare product, etc. }\end{array}$ & $\mathrm{PM}$ & $\begin{array}{l}\text { Fever, fatigue, loss of appetite, } \\
\text { jaundice, erythra, myalgia, } \\
\text { nausea and vomiting, ascites, } \\
\text { liver-palms, gray stool, } \\
\text { abdominal distension, etc. }\end{array}$ & $\begin{array}{l}\text { Mixed, } \\
\text { hepatocellular, } \\
\text { cholestasis }\end{array}$ & {$[7,14]$} \\
\hline
\end{tabular}

Note. PM means Polygonum multiflorum; the literature does not clearly indicate whether it is raw PM or PPM.

impaired the folding and processing of functional proteins, causing endoplasmic reticulum stress and generating liver cell apoptosis by repressing the biological control of the transcription and expression of peptidyl-prolyl cis-transisomerase A (PPIA) in a dose-dependent manner [57].

In addition, tannins induce the CYP2E1 enzyme [58], and previous studies concluded that the induction of CYP2E1 by tannin may affect the conversion and metabolism of AQs in vivo, which may lead to liver damage.

\subsection{TSG $\left(2,3,4^{\prime}, 5\right.$-Tetrahydroxystilbene-2-O- $\beta$-D-glucoside $)$} and Liver Injury. Stilbenes, especially TSG, are considered to be the main liver-protective components in PM. However, research has found that TSG might be associated with the hepatotoxicity of PM.

Generally, the adverse effect of PM will diminish or vanish, and it has tonifying effect after processing; in other words, the hepatotoxicity of raw PM is higher than that of PPM [21-25]. Coincidently, studies have found that the content of trans-TSG in PPM was reduced by $55.8 \%$ and the content of emodin in PPM was increased by $34 \%$ compared with that in PM; that is, the content of trans-TSG was reduced and the content of emodin was increased during the processing. Therefore, some reports speculated that the toxicity of PM might not be correlated with the content of emodin or its derivatives but depended on the contents of trans-TSG, conventionally thought to be a liver-protective compound, or the relative content of trans-TSG and emodin $[59,60]$. Reports have also shown that trans-TSG might inhibit the activation of the nuclear factor $\mathrm{kB}$ (NF-kB) signaling pathway, which is necessary for the HGF-mediated proliferation of WBF-344 cells. Trans-TSG may have some influence on the proliferation of liver cells [61-64].

Trans-TSG was unstable in irradiation and alkaline conditions and could convert to cis-TSG. Unfortunately, the latest research shows that cis-TSG has a higher cytotoxicity than trans-TSG in normal L02 cells, and cis-TSG could induce hepatotoxicity in LPS-treated rats. In addition, the contents of cis-TSG were higher in PM preparations or serum from patients with liver intoxication associated with PM than those in control samples, which may be because 
trans-TSG converted to cis-TSG in the course of preparation of PM and thus led to liver toxicity. All these results suggested that cis-TSG is closely associated with the hepatotoxicity of PM [65-67].

\section{Hepatotoxic Mechanism of PM}

In summary, the existing literature shows that the mechanism of PM-induced liver damage is mainly categorized into four viewpoints: (1) active substances in PM cause cholestasis, which leads to liver injury caused by lipid peroxidation or directly causes liver cell damage; (2) active substances in PM affect the CYP450 enzyme system, affecting drug transport or metabolism and leading to liver damage; (3) active substances in PM cause mitochondrial dysfunction, which leads to liver injury through the oxidative stress response; and (4) active substances in PM cause drug-induced liver injury (DILI).

3.1. Cholestasis and Liver Injury. Cholestasis and bile duct injury are the main clinical manifestations of cholestasis caused by chemical drugs. Serum biochemical parameters, including alkaline phosphatase (ALP), total bilirubin (TBIL), and bile acid (TBA), are preferred in clinical practice. Studies have shown that the ALP, TBIL, and TBA were significantly different in the serum of rats after administration with PM compared with the serum of control rats $[11,22,45]$, of which TBA increased in serum and increased in liver. Intrahepatic deposition of bile acids is considered to be the primary cause of cholestatic liver injury [68]. While cholic acid (CA), glycodeoxycholic acid (GDCA), chenodeoxycholic acid (CDCA), deoxycholic acid (DCA), and ursodeoxycholic acid were all significantly decreased, TCA was significantly increased, and GCA and GCDCA showed no significant changes [69]. These results indicated that the distribution of bile was also affected. The ethanol extract of PPM could affect the synthesis of bile acids and alter the composition of intestinal bile acids by activating Fxr-Fgf15 signaling in the intestine, and it can inhibit the expression of CYP7A1 in the liver [70]. In addition, AQs and dianthrones from PM, such as emodin and chrysophanol, significantly affected the activity of Bsep, Mrp2, and CYP8B1 and exhibited the strongest inhibitory effect on UGT1A1 activity, leading to bilirubin accumulation and resulting in cholestasis [34, 44-50, 71-73]. Meanwhile, hydrodeoxycholic acid (HDCA) in serum and tauro- $\beta$-muricholic acid (T $\beta$ MCA) in urine were identified in rats with $\mathrm{PM}$-induced liver injury [74-76]. Clinical analysis of drug-induced liver injury caused by PM or its preparations also indicated that there are many symptoms related to jaundice in patients with liver injury, including an increase in related indicators such as TBIL [7-10]. Therefore, liver injury caused by PM might be associated with cholestasis.

In the process of the generation, formation, transportation, and discharge of bile acid, failure at any step could cause cholestasis. When bile acid accumulation in liver cells or antioxidants decreases, the body will produces an oxidative stress reaction. This phenomenon could reduce the activity of superoxide dismutase (SOD), catalase (CAT), and glutathione peroxidase (GPx). First, ROS production increases; then, intestinal barrier dysfunction develops due to the lack of bile salts in the intestine. Next, excessive intestinal endotoxin (LPS) enters the systemic circulation, inducing the production of the inflammatory cytokines IL-1, IL-7, TNF- $\alpha$, etc. and generating additional ROS, which further aggravate oxidative damage in the body [76-78].

Excessive ROS in the body cause peroxidative damage in liver cells by directly damaging biomacromolecules such as proteins and DNA [79, 80]. ROS act as direct functional signaling molecules, activate intracellular stress-sensitive signaling pathways, and mediate hepatocyte apoptosis. ROS induce hepatocyte apoptosis by interfering with mitochondrial function and indirectly by activating apoptotic signaling pathways. In addition, ROS can mediate inflammatory reactions and then cause liver damage [81, 82], which explains the mechanisms of hepatitis symptoms in patients with PM-induced liver injury $[83,84]$.

3.2. CYP450s and Liver Injury. The CYP450 enzymes, which are highly concentrated and exhibit activities in the human liver, are responsible for the oxidation or reduction of medicines $[85,86]$. To date, more than 57 isoforms have been discovered in the CYP450 family; however, the CYP1A1, CYP1A2, CYP2B6, CYP2C8, CYP2C9, CYP2C19, CYP2D6, CYP2E1, CYP3A4, and CYP3A5 isoforms can metabolize nearly $90 \%$ of the drugs in the market [85]. It is well known that the activities of these CYP isoforms may be inhibited or induced by several compounds, and the modulation of their drug-metabolizing activities may have effects on pharmacodynamics and toxicology, such as DDI [86-88]. CYP450 and its isoenzymes are widely used in research fields such as drug metabolism, clinical rational drug use, toxicology, tumor biochemistry, and new drug research and development. In recent years, marked progress has been made in drug metabolism enzyme research.

Enzyme activity can be induced or inhibited by certain drugs to promote drug metabolism or to inhibit drug metabolism. If the metabolism of the drug inhibits the activity of a drug-metabolizing liver enzyme, the drug or its metabolites will accumulate in the liver and cause adverse reactions. The composition of traditional Chinese medicines is complex, but most TCM components are metabolized by the CYP450 enzyme and may further affect the metabolism or interaction of other drugs, such as the "eighteen incompatible medicaments," in which the combined application of Salvia, Sophora, Ginseng, and gourd inhibits the activity of CYP3A and CYP2E1 [89]. The combined administration of aconite, melon, Bletilla, Pinellia, Fritillaria, and Radix Ampelopsis inhibited the activity of CYP3A and CYP1A2 [90]. The activity of these metabolic enzymes is altered to impair the metabolism of the drug in the body, producing toxic effects.

3.2.1. CYP450 Inhibition. Research found that raw PM and PPM could significantly inhibit the expression of CYP2E1 mRNA [91]. The alcoholic extract of PM inhibited the activity of CYP2C19 and CYP2C9, and the aqueous extract of PM inhibited the activity of CYP2C19, CYP2C9, CYP2B6, 
CYP2D6, and CYP1A2 $[92,93]$. TSG has inhibitory effects on mouse liver CYP1A2, CYP2E1, and CYP3A11 protein expression through the suppression of AhR, PXR, and PPAR $\alpha$ activation [94]. CYP1A2, 2C19, and 2E1 have been reported as the main CYP450s which participated in phase I metabolism of AQs such as emodin [92, 95]. Therefore, it is speculated that the mechanism by which PM induces liver injury may be that some components in PM inhibit the activity of CYP2E1, which causes the accumulation of AQs or other toxic substances metabolized by CYP2E1, causing liver damage.

3.2.2. CYP450 Allelic Defect. The proportion of people with genetic defects in CYP450 enzymes is large [96]. However, due to the poor specificity of each CYP450 enzyme subtype substrate, each gene defect affects the metabolism of multiple drugs. Moreover, the incidence of different mutations that slow metabolism is different in different populations.

According to reports, whether CYP1A2 allele polymorphisms are associated with the acute liver injury induced by PM was tested by researchers using 43 cases of PM-induced liver injury. The results show that the frequency of the CYP1A21C allele was $46.5 \%$ in PM-induced DILI patients, which was significantly different from the frequency of $27.9 \%$ observed in healthy subjects. The frequency of the CYP1A21F allele was $63.9 \%$ in PM-induced DILI patients, compared to $57.0 \%$ in healthy controls; this difference was not significant. The allelic frequencies of CYP1A22, CYP1A27, CYP1A29, and CYP1A211 were not detected [97]. These results indicate that the CYP1A2 allelic mutation is most likely related to the metabolism of compounds in PM, followed by acute liver injury. Coincidentally, tannins, contained in rhubarb, are inducers of CYP2E1, which accelerate the conversion of $\mathrm{CCl}_{4}$ to $\mathrm{CCl}_{3}$, aggravating the liver injury caused by $\mathrm{CCl}_{4}$ [98]. Additionally, the activity of CYP450 may be related to the liver damage induced by PM.

\subsection{Mitochondrial Damage and Liver Injury.}

Mitochondria are important organelles that generate energy, oxidize materials in cells, and play a fundamental role in energy metabolism, free radical production, aging, and apoptotic regulation. Possible factors leading to mitochondrial dysfunction include respiratory chain defects, metabolic enzyme inactivation, structural changes, and mutations. All of these factors affect the normal function of cells, resulting in the occurrence of diseases. Mitochondria are important targets of drug toxicity, and mitochondria are also the organelles most vulnerable to drug toxicity. The liver is also a major target of drug damage because it is an important organ in drug metabolism. Drugs induce liver injury primarily by changing the activity of enzymes, modulating the structure of mitochondria and/or decreasing the synthesis of mtDNA, further undermining $\beta$-oxidation and other oxidative processes in liver cells [99-101].

Research shows that rhein could be changed into a reactive metabolite by CYP2C19, and the structure of reactive metabolite was inferred to be an epoxide compound. The reactive metabolite covalently binds to intracellular mitochondria, leading to ROS overproduction and respiratory chain dysfunction. In addition, the reactive metabolite overproduction depletes GSH, which could result in hepatotoxicity [102-104]. Research also found that emodin could conjugate with GSH, forming emodin-GSH and depleting GSH in PM-induced liver injury rats [40-42]. An increasing number of studies have shown that mitochondrial damage may be a factor and pathway that predisposes individuals to PM-induced liver injury.

\subsection{Drug-Induced Liver Injury}

3.4.1. Misuse of Counterfeit Goods. He Shou Wu, the roots of Polygonum multiflorum, was originally published in "Kai Bao Materia Medica" in the Northern Song Dynasty. This medicine has two kinds, red and white; the red type is commonly used in clinic. Common species used to as counterfeits of this medicine are Musa basjoo S. \& Z., Pteroxygonum giraldii Dammer \& Diels, Polygonum ciliinerve (Nakai) Ohwi, Stephania cepharantha Hayata, Cynanchum auriculatum Royle ex Wight, etc. In addition, PM has many origins, including Henan, Jiangxi, Guangdong, and Guangxi. The chemical content and efficacy of PM samples from different origins are also different [105-107]. Additionally, there is a large difference in quality. At present, due to insufficient market supervision, the confusion of origin and variety is also an important consideration in the safety of PM.

3.4.2. Improper Processing. Records on the processing of PM include the "Huatuo's Zhongzang Classic" in the eastern Han Dynasty, the "Chinese Pharmacopeia," national processing norms, and local processing norm. PM was primary processed in the pre-Tang Dynasty, steamed or cooked with black bean juice in the Tang Dynasty, and repeatedly steamed and sun-dried nine times in the Song and Qing Dynasty. The processing of PM has evolved from simple to complex, which indicates that people's understanding of the nourishing effect of PM continues to improve. The attenuating effects and efficiency of PM processing are constantly improving and have received increasing attention $[108,109]$. The continuous improvement of PM processing also indicates that there is a connection between the adverse reactions of PM and its processing. Modern research also proves that the toxicity of PM is significantly reduced after processing $[29,110-112]$. Therefore, processing is an important factor that impacts the effect of PM.

However, the processing of PM has changed from complicated to simple in modern times; for example, PM processing was simplified from nine cycles of steaming and sun-drying to steaming once without sun-drying. Additionally, the methods of PM processing vary among regions. There are many kinds of processing methods, including ancient processing and modern processing, such as boiling, steaming, exposure, frying, and roasting, which were without accessories; black beans, wine, vinegar, rice swill, and so on; nine cycles of steaming and sun-drying; high pressure processing, microwave processing, fermentation 
processing, extrusion technology, etc. $[109,113]$. Due to the diversity of processing norms, the types and contents of the chemical components in different processed products are different, which results in safety hazards in the clinical use of PM. The processing of PM could be combined with modern science and technology, such as the use of modern machinery and equipment (drum type frying machine, vacuum drying box) to achieve the homogeneity and stability of processing conditions. Furthermore, modern detection methods (HPLC, UV, GC, etc.) are used to achieve real-time quality monitoring.

In addition, researches have also speculated that the liver injury induced by PM might be related to an imbalance in intestinal flora [114], immune-specific effects, or idiosyncratic hepatotoxicity $[115,116]$.

3.4.3. Metals and Elements Were Associated with Liver Injury. During the process of planting medicinal materials, planting conditions such as sunlight, moisture, climate, and soil should be fully guaranteed. However, with the development of agriculture, organophosphorus pesticides and heavy metal residues have become a major environmental factor affecting the quality of Chinese herbal medicine. The heavy metals that cause environmental and soil pollution mainly include mercury, cadmium, lead, chromium, and arsenic.

Recent studies have also demonstrated that heavy metal and element concentrations are associated with PM-induced liver injury [117, 118]. Reports have also shown that heavy metal contamination may lead to adverse reaction [119]. PM has many kinds of habitats, and different habitats have different environmental conditions such as temperature and humidity. Thus, the components and inorganic elements, and even heavy metal residues of PM, are correlated to geographical, climatic, and soil factors $[120,121]$. Therefore, it is necessary to strengthen pharmacognosy traceability and quality control in order to prevent and reduce adverse reactions.

\section{Discussion}

According to the literature, the potential chemical composition and mechanism of liver injury caused by PM are summarized. The hepatotoxic compounds in PM (Figure 1) and the mechanism of the liver injury caused by PM need further study due to the integrity, complexity, and multitargeted and synergistic effects of this natural product.

First, as cholestasis in the body stimulates oxidative stress, the level of reactive oxygen species in the body increases, causing a series of reactions that lead to liver cell damage. Modern pharmacological studies have shown that many of the active ingredients in Chinese herbal medicines have antioxidation activity, and that they directly or indirectly reduce or eliminate the liver injury caused by cholestasis. For example, curcumin, a natural antioxidant in turmeric, plays an important role in the liver damage model caused by cholestasis by reducing the level of the inflammatory factor TNF- $\alpha$ in the liver $[122,123]$. Additionally, the active ingredients in herbs associated with liver injury have been reported to directly or indirectly cause oxidative damage to the body, resulting in hepatotoxicity, such as Melia toosendan, Dioscorea bulbifera L., and Radix Bupleuri $[124,125]$, and causing liver damage by inducing oxidative stress in the body. What is the mechanism of the liver injury caused by PM? The potential hepatotoxic components of PM can cause oxidative stress by destroying mitochondrial function, leading to cholestasis and then resulting in liver injury, or the potential hepatotoxic components of PM can affect the synthesis, transport, secretion, etc. of bile acid, causing cholestasis, which in turn disturbs oxidative balance in the body, leading to liver injury. These mechanisms need to be further studied.

Second, the clinical manifestation of liver injury caused by $\mathrm{PM}$ is the accumulation of bile acid in the liver. The accumulation of bile acid in the liver is considered to be the leading cause of cholestasis-induced liver injury. Any failure in the synthesis, transport, secretion, etc. of bile acids may cause cholestasis. There are two ways to synthesize bile acids in liver cells: the classic pathway, catalyzed by cholesterol $7 \alpha$-hydroxylase (CYP7A1), and the alternative pathway, catalyzed by sterol $27 \alpha$-hydroxylase. However, the alternative route is usually activated when the classic pathway is blocked. CYP3A is a key CYP450 enzyme that breaks down bile acids [126]. After bile acid is synthesized, it is secreted by active transport; approximately $95 \%$ of bile acids rely on a variety of transporters and energy to enter the liver and intestinal circulation. A variety of protein molecules with transport functions present on hepatocytes, bile duct cell membranes, and small intestinal cell membranes, such as Ntcp, Bsep Mrp2, Asbt, etc., directly affect the transport and secretion of bile acids [127]. Certain chemical constituents of PM function by inhibiting the expression of these transporters or by mutating these transporters, thus causing cholestasis [128]. In addition, as described above, cholestasis in vivo can induce an inflammatory response and the production of inflammatory factors. Studies have shown that intracellular LPS and some inflammatory factors can rapidly reduce the expression of Ntcp on hepatocytes, of which IL-1 is the most effective $[129,130]$. Notably, emodin and hydroxyemodin can inhibit the activity of Mrp2 according to reports. Is this the cause of liver damage in PM? Do the other medicinal substances in PM affect the activity of certain bile acid synthases or transport proteins; cause cholestasis in the synthesis, transport, and secretion of bile acids; and then promote liver injury?

Moreover, the degree of cholestasis-induced liver injury caused by PM processed with black bean was significantly decreased compared to that caused by PM without auxiliary materials (steamed PM). Additionally, steamed PM has a strong inhibitory effect on the activity of CYP2E1 [90, 131]. Black beans are common auxiliary materials in PM processing, and they are rich in protein, unsaturated fatty acids, vitamins, and phenolic acids, which have antioxidant and immunomodulatory effects $[132,133]$. PM processed with black bean has been used since ancient times and is included in the Pharmacopeia. However, whether changes in the chemical composition and content of PM occur after processing with black beans is uncertain. The mechanism of the positive effect of PM processed with black beans is unknown. 
<smiles>Cc1cc(O)c2c(c1)C(=O)c1cc(O)cc(O)c1C2=O</smiles>

Emodin<smiles>OC[C@H]1OC(Oc2c(O)cc(O)cc2/C=C/c2ccc(O)cc2)[C@H](O)[C@@H](O)[C@@H]1O</smiles>

THSG<smiles></smiles><smiles>O=C(OCC1O[C@@H](OC(=O)c2cc(O)c(O)c(OC(=O)c3cc(O)c(O)c(O)c3)c2)[C@H](OC(=O)c2cc(O)c(O)c(OC(=O)c3cc(O)c(O)c(O)c3)c2)[C@H](OC(=O)c2cc(O)c(O)c(OC(=O)c3cc(O)c(O)c(O)c3)c2)[C@@H]1OC(=O)c1cc(O)c(O)c(OC(=O)c2cc(O)c(O)c(O)c2)c1)c1cc(O)c(O)c(O)c1</smiles>

FIgURE 1: The structures of potential hepatotoxic compounds in PM.

Moreover, can black beans enhance the antioxidant activity of PM when PM is processed with black beans? Do the ingredients in black beans participate in the synthesis, transport, and secretion of bile acids? Is the attenuation of liver injury caused by PM exerted through these pathways? Is the mechanism of the positive effects of PM processed with black bean related to these factors? These questions require further study.

For example, as described in Section 1, researchers have speculated that the hepatotoxic compound in PM may be the emodin. However, emodin has strong antibacterial and antiinflammatory [124] activities [134] and inhibits the expression of inflammatory factors in the liver $[135,136]$. Additionally, emodin also has a certain antioxidant effect, which can reduce the level of oxidative stress in the liver and play a role in liver protection [137]. Researchers found that prepared rhubarb has a significant therapeutic effect on liver injury in animals, but it has certain hepatotoxic effects on normal animals. Thus, researchers have proposed the symptom-based prescription theory [138]. Based on the theory, the hepatotoxicity of PM was studied, and a high dosage of PM had a toxic effect on normal rats and a therapeutic effect on rats with chronic liver injury [139]. Thus, if emodin is the hepatotoxic component in PM, perhaps it is possible to study the hepatotoxic component in PM based on the symptom-based prescription theory.

\section{Conclusion}

PM has been widely used because of its tonifying function. There are 380 Chinese patent medicines containing PM. With the increasing number of clinical cases of adverse drug reactions caused by PM and its preparations, its safety issues have aroused great attention of domestic and foreign 
researchers and drug regulatory authorities. Thus, the liver injury of PM has become a practical problem that seriously affects the safety of its clinical medication and needs to be solved urgently. However, so far, there are still controversies about the liver damage components and mechanisms of PM. Research will be necessary for further understanding of the hepatotoxicity induced by PM so as to take reasonable and effective measures to prevent it. In addition, the clinical features of liver injury that result from PM include cholestasis hepatitis. Besides, the AQs in PM could interfere with bile acid metabolism according to the references. PM should be avoided in combination with drugs that can cause cholestatic liver injury such as chlorpromazine and rifampicin.

\section{Data Availability}

The data used to support the findings of this study are available from the corresponding author upon request.

\section{Conflicts of Interest}

The authors declare that they have no conflicts of interest regarding the publication of this paper.

\section{Authors' Contributions}

He-Shui Yu and Lin-Lin Wang contributed equally to this work.

\section{Acknowledgments}

This research was funded by the National Science and Technology Major Project of China (Grant no. 2018ZX09201011) and Science and Technology Program of Tianjin Municipal Commission of Education (Grant no. 2019KJ079).

\section{References}

[1] Chinese Pharmacopoeia Commission, Pharmacopoeia of the People's Republic of China, Beijing: Chemical Industry Press, vol. 1, 2020.

[2] T.-T. Ho, H. N. Murthy, D. Dalawai, M. A. Bhat, K.-Y. Paek, and S.-Y. Park, "Attributes of Polygonum multiflorum to transfigure red biotechnology," Applied Microbiology and Biotechnology, vol. 103, no. 8, pp. 3317-3326, 2019.

[3] L. Lin, B. Ni, H. Lin et al., "Traditional usages, botany, phytochemistry, pharmacology and toxicology of Polygonum multiflorum Thunb.: a review," Journal of Ethnopharmacology, vol. 159, pp. 158-183, 2015.

[4] H. Dong, D. Slain, J. Cheng, W. Ma, and W. Liang, "Eighteen cases of liver injury following ingestion of Polygonum multiflorum," Complementary Therapies in Medicine, vol. 22, no. 1, pp. 70-74, 2014.

[5] K. A. Jung, H. J. Min, S. S. Yoo et al., "Drug-induced liver injury: twenty five cases of acute hepatitis following ingestion of Polygonum multiflorum thunb," Gut and Liver, vol. 5, no. 4, pp. 493-499, 2011.

[6] J.-B. Yang, W.-F. Li, Y. Liu et al., "Acute toxicity screening of different extractions, components and constituents of Polygonum multiflorum Thunb. on zebrafish (Danio rerio) embryos in vivo," Biomedicine \& Pharmacotherapy, vol. 99, pp. 205-213, 2018.

[7] H.-Z. Wang and X.-H. Li, "Clinical analysis of 33 cases of drug induced liver injury caused by Polygonum multiflorum Thunb and its preparations," Chinese Journal of Integrated Traditional and Western Medicine on Liver Diseases, vol. 28, no. 1, pp. 25-27, 2018.

[8] Y. Zhu, Y.-G. Li, Y. Wang et al., "Analysis of clinical characteristics in 595 patients with Herb-induced liver injury," Chinese Journal of Integrated Traditional and Western Medicine, vol. 36, pp. 44-48, 2016.

[9] L. Xiang, T.-T. Ke, and A.-R. Hu, "Clinical analysis of 52 patients with liver injury induced by Ploygonum multiflorum and its preparation," Chinese Archives of Traditional Chinese Medicine, vol. 31, pp. 1133-1134, 2013.

[10] Y. Zhu, S.-H. Liu, J.-B. Wang et al., "Clinical analysis of druginduced liver injury caused by Polygonum multiflorum and its preparations," Chinese Journal of Integrated Traditional and Western Medicine, vol. 35, no. 12, pp. 1442-1447, 2015.

[11] Y. Zhang, S. Chen, and L. Lu, "Clinical analysis of 36 cases of drug-induced liver injury by Polygonum multiflorum Thunb," Hainan Medical Journal, vol. 24, no. 2, pp. 235-237, 2013.

[12] Q.-B. Fu, T.-C. Liu, Y.-Z. Yu, F.-X. Xiao, and Z. Zhou, "Epidemiological and clinical characteristics of 140 cases of drug-induced liver injury caused by Polygonum multiflorum and its preparations," Chinese Journal of Integrated Traditional and Western Medicine on Liver Diseases, vol. 30, no. 1, pp. 6-9, 2020.

[13] X.-J. Xie, K.-F. Ma, and Y. Liu, "Case analysis of liver injury induced by Polygoni multiflori radix case analysis of liver injury induced by Polygoni multiflori radix," Drug Evaluation, vol. 9, no. 32, pp. 36-38, 2012.

[14] Y. Chang, Q.-Y. Liu, C.-J. Lv, Y.-P. Ding, W. Zhang, and H. Li, "Clinical features of 39 patients with acute liver injury induced by Polygonum multiflorum," Chinese Hepatology, vol. 23, no. 8, pp. 670-672, 2018.

[15] L. Wang, M. Sang, E. Liu et al., "Rapid profiling and pharmacokinetic studies of major compounds in crude extract from Polygonum multiflorum by UHPLC-Q-TOF-MS and UPLC-MS/MS," Journal of Pharmaceutical and Biomedical Analysis, vol. 140, pp. 45-61, 2017.

[16] J.-B. Yang, Y. Liu, Q. Wang et al., "Characterization and identification of the chemical constituents of Polygonum multiflorum Thunb. by high-performance liquid chromatography coupled with ultraviolet detection and linear ion trap FT-ICR hybrid mass spectrometry," Journal of Pharmaceutical and Biomedical Analysis, vol. 172, pp. 149-166, 2019.

[17] Y. Liu, Q. Wang, J.-B. Yang et al., "Polygonum multiflorum thunb: a review on chemical analysis, processing mechanism, quality evaluation, and hepatotoxicity," Frontiers in Pharmacology, vol. 9, p. 364, 2018.

[18] R. Zhang, B. Liu, Z.-X. Sun, and D.-Y. Xu, "Effects of extract of Polygonum multiflorum on cell cycle arrest and apoptosis of human liver cell line L02," Journal of Chinese Integrative Medicine, vol. 8, no. 6, pp. 554-561, 2010.

[19] Z.-Z. Ge, C. Zhang, G.-Y. Feng, H.-L. Yang, L.-Y. Shi, and Z.-X. Sun, "Study on acute hepatotoxicity of Polygonum multiflorum extract in normal and hepatocarcinoma-bearing mice," China Pharmacy, vol. 25, pp. 1358-1360, 2014.

[20] T. Wang, J.-Y. Wang, Z.-X. Zhou, Y.-Y. Li, L. Zhang, and L.-P. Zhang, "Study on hepatotoxicity of aqueous extracts of Polygonum multiflorum in rats after 28-day oral 
administration: choleatasis-related mechanism," China Journal of Chinese Materia Medica, vol. 40, pp. 2163-2167, 2015.

[21] C. Tu, B.-Q. Jiang, Y.-L. Zhao et al., "Comparison of processed and crude Polygoni multiflori radix induced rat liver injury and screening for sensitive indicators," China Journal of Chinese Materia Medica, vol. 40, pp. 654-660, 2015.

[22] Q. Li, K.-J. Zhao, Y.-L. Zhao et al., "High dosage administration of Polygonum multiflorum alcohol extract caused the multi-organ injury," Global Traditional Chinese Medicine, vol. 6, pp. 1-7, 2013.

[23] W. Huang, Y.-N. Zhang, and R. Sun, "Experimental study on the "Dose-Time-Toxicity" relationship of acute hepatotoxicity induced by different components from Polygonum multiflorum in Mice," Chinese Journal of Pharmacovigilance, vol. 18, pp. 193-197, 2011.

[24] T. Noda, T. Yamada, T. Ohkubo et al., "Hot-water-extracts of Polygonum multiflorum do not induce any toxicity but elicit limited beneficial effects on the liver in mice," Journal of Health Science, vol. 55, no. 5, pp. 720-725, 2009.

[25] T. Wang, J.-Y. Wang, Z.-Z. Jiang et al., "Study on hepatotoxicity of aqueous extracts of Polygonum multiflorum in rats after 28-day oral administration-analysis on correlation of cholestasis," China Journal of Chinese Materia Medica, vol. 37, pp. 1445-1450, 2012.

[26] X.-B. Sun, Y.-W. Sun, H. Li, and W. Sun, "Influence of main component of Heshouwu such as emodin, rhein and toluylene glycoside on hepatic cells and hepatoma carcinoma cell," Modern Journal of Integrated Traditional Chinese and Western Medicine, vol. 19, pp. 1315-1319, 2010.

[27] M. Zhang, L.-F. Lin, H.-M. Lin, C.-H. Qu, L. Yan, and J. Ni, "Interpretation the hepatotoxicity based on pharmacokinetics investigated through oral administrated different extraction parts of Polygonum multiflorum on rats," Frontiers in Pharmacology, vol. 9, p. 505, 2018.

[28] L. Lin, H. Lin, M. Zhang et al., "A novel method to analyze hepatotoxic components in Polygonum multiflorum using ultra-performance liquid chromatography-quadrupole timeof-flight mass spectrometry florum using ultra-performance liquid chromatography-quadrupole time-of-flight mass spectrometry," Journal of Hazardous Materials, vol. 299, pp. 249-259, 2015.

[29] G. P. Lv, L. Z. Meng, D. Q. Han, H. Y. Li, J. Zhao, and S. P. Li, "Effect of sample preparation on components and liver toxicity of Polygonum," Journal of Pharmaceutical and Biomedical Analysis, vol. 109, pp. 105-111, 2015.

[30] W. Rui, W. Xia, W. Zhao et al., "Differential constituents in roots, stems and leaves of Polygonum multiflorum thunb. screened by UPLC/ESI-Q-TOF-MS and multivariate statistical analysis," Journal of Chromatographic Science, vol. 58, no. 2, pp. 136-143, 2020.

[31] J. Yu, J. Xie, X.-J. Mao et al., "Hepatoxicity of major constituents and extractions of radix polygoni multiflori and radix polygoni multiflori praeparata," Journal of Ethnopharmacology, vol. 137, no. 3, pp. 1291-1299, 2011.

[32] B. Zhang, S.-H. Din, X.-M. Qian, and W.-S. Zhu, "Q Lu, "Liver function of drug-induced liver injury induced by emodin in rats," Military Medical Journal of South China, vol. 29, pp. 735-738, 2015.

[33] M.-X. Wang, Y.-G. Wang, H.-H. Xu et al., "Effects of emodin in Polygonum multiflorum on liver cytotoxicity and CYP450 isoenzymes expression in L02 cells," Chinese Pharmacological Bulletin, vol. 32, pp. 1543-1548, 2016.
[34] Q. Wang, Z. Dai, Y.-J. Zhang, and S.-C. Ma, "Study on the hepatotoxicity of dianthrones in Polygoni multiflori radix," Chinese Journal of Pharmaceutical Analysis, vol. 38, pp. 268-274, 2018.

[35] J. Ma, L. Zheng, Y.-S. He, and H.-J. Li, "Hepatotoxic assessment of Polygoni multiflori Radix extract and toxicokinetic study of stilbene glucoside and anthraquinones in rats," Journal of Ethnopharmacology, vol. 162, no. 13, pp. 61-68, 2015.

[36] L. F. Han, P. Wang, Y. L. Wang et al., "Rapid discovery of the potential toxic compounds in Polygonum multiflorum by UHPLC/Q-Orbitrap-MS-Based metabolomics and correlation analysis," Frontiers in Pharmacology, vol. 10, p. 329, 2019.

[37] D.-Q. Luo, P. Jia, S. S. Zhao et al., "Identification and differentiation of Polygonum multiflorum Radix and Polygoni multiflori Radix preaparata through the quantitative analysis of multicomponents by the single-marker method," Journal of Analytical Methods in Chemistry, vol. 2019, Article ID 7430717, 2019.

[38] T.-T. A. Nguyen, M. T. Ha, S.-E. Park, J. S. Choi, B. S. Min, and J. A. Kim, "Stilbenes with potent protein tyrosine phosphatase-1B inhibitory activity from the roots of Polygonum multiflorum," Journal of Natural Products, vol. 83, no. 2, pp. 323-332, 2020.

[39] L. Zhang, D. He, K. Li et al., "Emodin targets mitochondrial cyclophilin D to induce apoptosis in HepG2 cells," Biomedicine \& Pharmacotherapy, vol. 90, pp. 222-228, 2017.

[40] L.-L. Jiang, D.-S. Zhao, Y.-X. Fan, Q. Yu, P. Li, and H.-J. Li, "Detection of emodin derived glutathione adduct in normal rats administered with large dosage of Polygoni multiflori Radix," Frontiers in Pharmacology, vol. 8, p. 446, 2017.

[41] D.-M. Liu, C.-Y. Zhou, J.-S. Wu, P. Wang, and X.-L. Meng, "Emodin induces apoptosis in HepG2 cells via mitochndrial pathway," Chinese Journal of Experimental Traditional Medical Formulae, vol. 24, no. 3, pp. 104-108, 2018.

[42] D.-M. Liu, C.-Y. Zhou, J.-S. Wu, P. Wang, and X.-L. Meng, "Emodin induces apoptosis of L02 via caspase-8-mediated activation of the mitochondrial death pathway," Pharmacology and Clinics of Chinese Materia Medica, vol. 33, no. 5, pp. 23-26, 2017.

[43] L. Lin, Y. Liu, S. Fu, C. Qu, H. Li, and J. Ni, "Inhibition of mitochondrial complex function-the hepatotoxicity mechanism of emodin based on quantitative proteomic analyses," Cells, vol. 8, no. 3, p. 263, 2019.

[44] L. Kang, L. Si, J. Rao et al., "Polygoni Multiflori Radix derived anthraquinones alter bile acid disposition in sandwich-cultured rat hepatocytes," Toxicology in Vitro, vol. 40, pp. 313-323, 2017.

[45] Q. Wang, Y.-D. Wang, Y. Li et al., "Identifcation and characterization of the structure-activity relationships involved in UGT1A1 inhibition by anthraquinone and dianthrone constituents of Polygonum multiforum," Scientific Reports, vol. 7, no. 1, Article ID 17952, 2017.

[46] M.-J. Wei, J. Huang, J.-Q. Bai, X.-C. Cai, and X.-H. Qiu, "Effects of emodin on serum liver function, liver transporter and metabolic enzyme UGT1A1 expression in rats," Lishizhen Medicine and Materia Medica Research, vol. 29, no. 7, pp. 1551-1555, 2018.

[47] J. Seppen, P. J. Bosma, B. G. Goldhoorn et al., "Discrimination between Crigler-Najjar type I and II by expression of mutant bilirubin uridine diphosphate-glucuronosyltransferase," Journal of Clinical Investigation, vol. 94, no. 6, pp. 2385-2391, 1994. 
[48] M. G. Mustafa, M. L. Cowger, and T. E. King, "Effects of bilirubin on mitochondrial reactions," The Journal of Biological Chemistry, vol. 244, no. 23, pp. 6403-6414, 1969.

[49] M. L. Cowger, "Mechanism of bilirubin toxicity on tissue culture cells: factors that affect toxicity, reversibility by albumin, and comparison with other respiratory poisons and surfactants," Biochemical Medicine, vol. 5, no. 1, pp. 1-16, 1971.

[50] E. Sticova and M. Jirsa, "New insights in bilirubin metabolism and their clinical implications," World Journal of Gastroenterology, vol. 19, no. 38, pp. 6398-6307, 2013.

[51] M.-X. Wang, Study on the Mechanisms of Emodin Increasing Liver Injury in Polygonum Multiflorum Thunb through Activating CYP1A1, Guangdong Pharmaceutical University, Guangzhou, China, 2018.

[52] W. Liu, Metabolism of Emodin in Liver and Intestine and its Gender-dependent Differences, Southern Medical University, Guangzhou, China, 2010.

[53] M. Yang, T. Liu, W.-H. Feng et al., "Exploration research on hepatotoxic constituents from Polygonum multiflorum root," China Journal of Chinese Materia Medica, vol. 41, no. 10, pp. 1289-1296, 2016.

[54] Z.-L. Liu, Z.-Q. Song, L. Zhang, and S.-L. Li, "Influence of process methods on contents of chemical component radix Polygoni multiflori," China Journal of Chinese Materia Medica, vol. 30, no. 5, pp. 336-340, 2005.

[55] X. Zhou, W.-J. Luo, L.-P. Wang, and P.-F. Wei, "Study on the toxic mechanism of different components of Polygonum multiflorum Thunb," Journal of Liaoning University of Traditional Chinese Medicine, vol. 21, no. 1, pp. 51-53, 2019.

[56] X.-Q. Hu, Y.-L. Li, and L. Wang, "Effect of tannin in Polygonum multiflorum on liver biochemical indexes of rats," Drug Evaluation Research, vol. 33, no. 1, pp. 63-65, 2010.

[57] Y. Wu, Screening of in Vitro Model in Drug-Induced Liver Injury and Preliminary in Vestigation of Polygonum Multiflorum Induced Liver Injury, Chinese Academy of Medical Sciences \& China Union Medical College, Beijing, China, 2016.

[58] R.-B. Huang, L.-Q. Zang, G. Liang, X. Lin, and N.-P. Wang, "Effect of EGCG on hepatic microsomal drug-metabolizing enzyme and CCl4-induced liver injury," Journal of Guangxi University of Chinese Medicine, vol. 16, no. 3, pp. 129-131, 1999.

[59] X. Wu, X. Chen, Q. Huang, D. Fang, G. Li, and G. Zhang, "Toxicity of raw and processed roots of Polygonum multiflorum," Fitoterapia, vol. 83, no. 3, pp. 469-475, 2012.

[60] Y.-Q. Bao, F. Shen, Y.-L. Li, D.-M. Chen, and H. Lu, "Toxicity and mechanism of Polygoni multiflori radix alcohol extract on L02 cells," Chinese Journal of Experimental Traditional Medical Formulae, vol. 26, no. 10, pp. 23-28, 2020.

[61] X.-Q. Hu, M. Lin, H.-L. Yang et al., "Effects of different ratios of Tannins and Stilbene glucoside from Polygonum multiflorum on liver biochemical indexes in rats," Shanghai Journal of Traditional Chinese Medicine, vol. 45, no. 4, pp. 56-59, 2011.

[62] P. Yao and D.-R. Hu, "Role of NF-kB in HGF-mediated proliferation of WBF-344 cell," Medical Journal of Chinese People's Liberation Army, vol. 34, no. 3, pp. 271-273, 2009.

[63] S.-Y. Long, C.-P. Zhang, X.-Q. Gao et al., "Effects of 2,3,5,4'tetrahydroxystilbene-2-O- $\beta$-D-glucoside on the expression of NF-kB/IkB and apoptosis of HUVECs induced by $\mathrm{H}_{2} \mathrm{O}_{2}$," Chinese Pharmacological Bulletin, vol. 27, no. 10, pp. 13531357, 2011.
[64] R.-C. Zhang, C. Zhang, Z.-X. Sun, and Q.-H. Deng, "Damage effect of Polygonum multiflorum fractions on human normal liver cells L02 and liver cancer cells HepG2," China Journal of Chinese Materia Medica, vol. 37, no. 12, pp. 1830-1835, 2012.

[65] C. Li, M. Niu, Z. Bai et al., "Screening for main components associated with the idiosyncratic hepatotoxicity of a tonic herb, Polygonum multiflorum," Frontiers of Medicine, vol. 11, no. 2, pp. 253-265, 2017.

[66] X.-L. Xu, J. Ren, W.-Z. Zhai et al., "Evaluation of hepatotoxicity of different parts of Polygonum multiflorum and Polygonum multiflorum by HPLC fingerprint," Chinese Traditional Patent Medicine, vol. 42, no. 3, pp. 792-795, 2020.

[67] Y.-K. Meng, C.-Y. Li, R.-Y. Li et al., "Cis-stilbene glucoside in Polygonum multiflorum induces immunological idiosyncratic hepatotoxicity in LPS-treated rats by suppressing PPAR- $\gamma$," Acta Pharmacologica Sinica, vol. 38, no. 10, pp. 1340-1352, 2017.

[68] B. Copple, H. Jaeschke, and C. Klaassen, "Oxidative stress and the pathogenesis of cholestasis," Seminars in Liver Disease, vol. 30, no. 02, pp. 195-204, 2010.

[69] Z.-J. Ma, The Initial Study of Objectivity, Clinical Metabolic Biomarker and Injury Mechanism of Hepatotoxicity of Polygoni Multiflori Radix, Chengdu University of TCM, Chengdu, China, 2013.

[70] J. Wei, J. Chen, L. Fu et al., "Polygonum multiflorum Thunb suppress bile acid synthesis by activating Fxr-Fgf15 signaling in the intestine," Journal of Ethnopharmacology, vol. 235, pp. 472-480, 2019.

[71] Z.-F. Tang, G. Ma, and Q.-X. Mei, "Effect of bilirubin on hepatocyte uptake and bile excretion before and after processing of Polygonum multiflorum," Lishizhen Medicine and Materia Medica Research, vol. 29, no. 3, pp. 595-598, 2018.

[72] X. Wang, L. Han, Y. Bi et al., "Paradoxical effects of emodin on ANIT-induced intrahepatic cholestasis and herb-induced hepatotoxicity in mice," Toxicological Sciences, vol. 168, no. 1, pp. 264-278, 2019.

[73] L. Wu, W. Han, Y. Chen et al., "Gender differences in the hepatotoxicity and toxicokinetics of emodin: the potential mechanisms mediated by UGT2B7 and MRP2," Molecular Pharmaceutics, vol. 15, no. 9, pp. 3931-3945, 2018.

[74] X. Jing, C.-Q. Wu, G. Han et al., "Study on cholestasis and related protein express in rats with liver injury induced by Polygonum multiflorum," Drug Evaluation Research, vol. 40, no. 5, pp. 612-619, 2017.

[75] D.-S. Zhao, L.-L. Jiang, Y.-X. Fan et al., "Identification of urine tauro- $\beta$-muricholic acid as a promising biomarker in Polygoni multiflori radix-induced hepatotoxicity by targeted metabolomics of bile acids," Food and Chemical Toxicology, vol. 108, pp. 532-542, 2017.

[76] N. Singh and L. Li, "Reduced oxidative tissue damage during endotoxemia in IRAK-1 deficient mice," Molecular Immunology, vol. 50, no. 4, pp. 244-252, 2012.

[77] D. Weber-Mzell, P. Zaupa, T. Petnehazy et al., "The role of nuclear factor-kappa B in bacterial translocation in cholestatic rats," Pediatric Surgery International, vol. 22, no. 1, pp. 43-49, 2006.

[78] H. Jaeschke, "Reactive oxygen and mechanisms of inflammatory liver injury: present concepts," Journal of Gastroenterology and Hepatology, vol. 26, pp. 173-179, 2011.

[79] K. Allen, H. Jaeschke, and B. L. Copple, "Bile acids induce inflammatory genes in hepatocytes," The American Journal of Pathology, vol. 178, no. 1, pp. 175-186, 2011. 
[80] M. J. Vallejo, L. Salazar, and M. Grijalva, "Oxidative stress modulation and ROS-mediated toxicity in cancer: a review on in vitro models for plant-derived compounds," Oxidative Medicine and Cellular Longevity, vol. 2017, Article ID 4586068, 2017.

[81] C.-X. Shang, Y.-B. Chen, L. Heng et al., "Damage to mtDNA in liver injury of patients with extrahepatic cholestasis: the protective effects of mitochondrial transcription Factor A," Free Radical Biology and Medicine, vol. 52, no. 9, pp. 1543-1551, 2012.

[82] E. Oqlodek, B. Augustyńska, A. Araszkiewicz, and D. Moś, "The bile acids as an example of patogens destructive hepatocytes in alcoholic liver injury," Polski Merkuriusz Lekarski, vol. 27, no. 160, pp. 346-348, 2009.

[83] S.-L. Yang, "Clinical analysis of 30 cases of drug-induced hepatitis caused by fallopia multiflora," Chinese Community Doctors, vol. 34, no. 28, pp. 118-120, 2018.

[84] G. Mazzanti, L. Battineli, C. Daniele et al., "New case of acute hepatitis following the consumption of Shou Wu Pian, a Chinese herbal product derived from Polygonum multiflorum," Annals of Internal Medicine, vol. 140, no. 7, Article ID IDW30, 2004.

[85] P. R. Ortiz de Montellano and J. J. De Voss, "Oxidizing species in the mechanism of cytochrome P450," Natural Product Reports, vol. 19, no. 4, pp. 477-493, 2002.

[86] U. M. Zanger and M. Schwab, "Cytochrome P450 enzymes in drug metabolism: regulation of gene expression, enzyme activities, and impact of genetic variation," Pharmacology \& Therapeutics, vol. 138, no. 1, pp. 103-141, 2013.

[87] C. Inglese, M. Grazia Perrone, F. Berardi, R. Perrone, and N. Antonio Colabufo, "Modulation and absorption of xenobiotics: the synergistic role of CYP450 and P-gp activities in cancer and neurodegenerative disorders," Current Drug Metabolism, vol. 12, no. 8, pp. 702-712, 2011.

[88] Y. Masubuchi and T. Horie, "Toxicological significance of mechanism-based inactivation of cytochrome p450 enzymes by drugs," Critical Reviews in Toxicology, vol. 37, no. 5, pp. 389-412, 2007.

[89] H.-Y. Dong, J.-W. Shao, J.-F. Chen, T. Wang, F.-P. Lin, and Y.-H. Guo, "Transcriptional regulation of cytochrome P450 3A4 by four kinds of traditional Chinese medicines," China Journal of Chinese Materia Medica, vol. 33, no. 9, pp. 1014-1017, 2008.

[90] K.-T. Jin, Y.-G. Wang, S.-Y. Shi, J.-X. Sheng, and Y. Gao, "Interaction between radix aconiti and rhizoma bletillae based on cytochrome P450 in rat livers," China Journal of Traditional Chinese Medicine and Pharmacy, vol. 22, pp. 598-602, 2007.

[91] P.-F. Wei, M. Zhang, C.-L. Jiao, and Y.-Y. Wu, "Effect of different radix Polygoni multiflori preparata on CYP2E1 mRNA expression in rat liver," Chinese Journal of Hospital Pharmacy, vol. 30, no. 17, pp. 1445-1448, 2010.

[92] Y. Zhang, T. Ding, T. Diao, M. Deng, and S. Chen, "Effects of Polygonum multiflorum on the activity of cytochrome P450 isoforms in rats," Die Pharmazie, vol. 70, no. 1, pp. 47-54, 2015.

[93] H. Li, H.-L. Yang, D.-K. Li et al., "Study on inhibitory effect of water extract of Polygonum multiflorum on CYP1A2 and CYP2E1 enzymatic activities and mRNA expressions in rat liver," China Journal of Chinese Materia Medica, vol. 40, no. 7, pp. 1370-1375, 2015.

[94] Y.-Y. Wang, J. Yang, H. Liu, F.-Q. Lin, J.-S. Shi, and F. Zhang, "Effects of tetrahydroxystilbene glucoside on mouse liver cytochrome P450 enzyme expressions," Xenobiotica, vol. 45, no. 4, pp. 279-285, 2015.

[95] L.-L. Ouyang, W. Xu, J.-Q. Bai, L. Guo, and X.-H. Qiu, "In vitro metabolism of emodin in human liver microsomes," Guiding Journal of Traditional Chinese Medicine and Pharmacy, vol. 21, pp. 14-18, 2015.

[96] A. K. Daly, "Genetic polymorphisms affecting drug metabolism," Current Concepts in Drug Metabolism and Toxicology, vol. 63, pp. 137-167, 2012.

[97] K. F. Ma, X. G. Zhang, and H. Y. Jia, "CYP1A2 polymorphism in Chinese patients with acute liver injury induced by Polygonum multiflorum," Genetics and Molecular Research, vol. 13, no. 3, pp. 5637-5643, 2014

[98] J.-B. Wang, Y.-G. Ma, P. Zhang et al., "Effect of processing on the chemical contents and hepatic and renal toxicity of rhubarb studied by canonical correlation analysis," Acta Pharmaceutica Sinica, vol. 44, no. 8, pp. 885-890, 2009.

[99] T.-T. Yang, Z.-Z. Jiang, and L.-Y. Zhang, "Research progress in drug-induced liver injury via mitochondrial damage," Progress in Pharmaceutical Sciences, vol. 38, no. 11, pp. 809-818, 2014.

[100] M. P. C. Ribeiro, A. E. Santos, and J. B. A. Custódio, "Mitochondria: the gateway for tamoxifen-induced liver injury," Toxicology, vol. 323, pp. 10-18, 2014.

[101] I. Shokolenko, N. Venediktova, A. Bochkareva, G. L. Wilson, and M. F. Alexeyev, "Oxidative stress induces degradation of mitochondrial DNA," Nucleic Acids Research, vol. 37, no. 8, pp. 2539-2548, 2009.

[102] L.-N. He, A.-H. Yang, T.-Y. Cui et al., "Reactive metabolite activation by CYP2C19-mediated rhein hepatotoxicity," Xenobiotica, vol. 45, no. 4, pp. 361-372, 2015.

[103] D. Bironaite and K. Öllinger, "The hepatotoxicity of rhein involves impairment of mitochondrial functions," Chemicobiological Interactions, vol. 103, no. 1, pp. 35-50, 1997.

[104] Q. Du, X.-L. Bian, X.-L. Xu, B. Zhu, B. Yu, and Q. Zhai, "Role of mitochondrial permeability transition in human hepatocellular carcinoma Hep-G2 cell death induced by rhein," Fitoterapia, vol. 91, no. 6, pp. 68-73, 2013.

[105] C. Ma, H.-J. Yan, C.-P. Tang, and Z.-J. Fang, "Study on antioxidant effects of intragastric administration with water decoction of Polygonum multiflorum thumb from different provenances on aging mice," Journal of Guangdong College of Pharmacy, vol. 24, no. 6, pp. 581-583, 2008.

[106] Y.-Y. Luo, J.-X. Liu, X.-H. Liu et al., "Difference of chemical compositions in Polygoni multifori radix from different habitats by UPLC-Triple TOF MS/MS," Journal of Chinese Mass Spectrometry Society, vol. 38, no. 6, pp. 679-689, 2017.

[107] Y. Chen, The Study of Quality Evaluation and Regional Division of Polygonum Multiflorum Thunb, Guangzhou University of Chinese Medicine, Guangdong, China, 2013.

[108] H. Gu and Y. Zhang, "A study on ancient and modern preparation of Heshouwu," Clinical Journal of Chinese Medicine, vol. 7, no. 34, pp. 24-26, 2015.

[109] H.-R. Cui, Z.-F. Bai, H.-B. Song, T.-Z. Jia, J.-B. Wang, and X.-H. Xiao, "Investigation of potential toxic factors for fleece-flower root: from perspective of processing methods evolution," China Journal of Chinese Materia Medica, vol. 41, no. 2, pp. 333-339, 2016.

[110] Y.-M. Zhou, M.-J. Zhao, X.-H. Gong et al., "The influence of different processing time on composition and content of Polygoni multiflori," Natural Product Research and Development, vol. 29, no. 10, pp. 1759-1765, 2017.

[111] X.-M. Zhao, X.-Y. Li, R. Sun et al., "Study on acute toxicity of different processed ethanol extract of Polygoni multiflori in 
mice," Chinese Journal of Pharmacovigilance, vol. 14, no. 10, pp. 603-610, 2017.

[112] D. Gao, X.-F. Li, P. Yin et al., "Preliminary study on hepatotoxic components in Polygoni multiflori radix based on processing and toxicity-decreasing," Chinese Traditional and Herbal Drugs, vol. 48, no. 10, pp. 2044-2050, 2017.

[113] R. Zou, J.-J. Xiao, Y.-Y. Wang, and Y.-X. Wu, "Brief description of processing technology of Polygoni multiflori Radix; a study on ancient and modern preparation of heshouwu," Guangzhou Chemical Industry, vol. 48, no. 7, pp. 21-23, 2020.

[114] H. Zheng, Study on Gut Microbiota Changes in Polygonum Multiflorum Thunb. Induced Liver Injury Rats by HighThroughput Sequencing and Real-Time Quantitative PCR, Guangdong Pharmaceutical University, Guangdong, China, 2017.

[115] L.-Z. He, P. Yin, Y.-K. Meng et al., "Study on the mechanism of PPAR- $\gamma$ dependent immunological idiosyncrasy liver injury induced by Polygonum multiflorum," Acta Pharmaceutica Sinica, vol. 52, no. 7, pp. 1027-1032, 2017.

[116] C.-P. Li, T. Rao, X.-P. Chen et al., "HLA-B*35:01 allele is a potential biomarker for predicting polygonummultifloruminduced liver injury in humans," Hepatology, vol. 70, no. 1, pp. 346-357, 2019.

[117] E. J. Werder, J. I. Beier, D. P. Sandler et al., "Blood BTEXS and heavy metal levels are associated with liver injury and systemic inflammation in Gulf states residents," Food and Chemical Toxicology, vol. 139, Article ID 111242, 2020.

[118] M. Şahin, F. Karayakar, K. E. Erdogan, F. Bas, and T. Colak, "Liver tissue trace element levels in HepB patients and the relationship of these elements with histological injury in the liver and with clinical parameters," Journal of Trace Elements in Medicine and Biology:Organ of the Society for Minerals and Trace Elements (GMS), vol. 45, pp. 70-77, 2018.

[119] J. Wang, Z. Ma, M. Niu et al., "Evidence chain-based causality identification in herb-induced liver injury: exemplification of a well-known liver-restorative herb Polygonum multiflorum," Frontiers of Medicine, vol. 9, no. 4, pp. 457-467, 2015.

[120] L. Xin, L. Meng, S.-L. Wei, X.-J. Qi, and B.-Y. Zhuo, "Analysis on functional components of Polygonum multiflorum from different habitats," Modern Chinese Medicine, vol. 22, no. 3, pp. 384-390, 2020.

[121] J. Yang, L. Zhou, L. Hu et al., "Quantitative analysis of 40 inorganic elements in Polygoni multiflori radix from different origins by ICP-MS," World Chinese Medicine, vol. 14, no. 11, pp. 2819-2828, 2019.

[122] Y.-Y. Yang, K.-C. Lee, Y.-T. Huang et al., "Effects of $\mathrm{N}$-acetylcysteine administration in hepatic microcirculation of rats with biliary cirrhosis," Journal of Hepatology, vol. 49, no. 1, pp. 25-33, 2008.

[123] T. Y. Lee, F.-Y. Chen, H.-H. Chang, and H.-C. Lin, "The effect of capillarisin on glycochenodeoxycholic acid-induced apoptosis and heme oxyge-nase- 1 in rat primary hepatocytes," Molecular and Cellular Biochemistry, vol. 325, no. 1-2, pp. 53-59, 2009.

[124] S.-Y. Qi, R.-M. Jin, H.-J. Liu, and Y.-W. Huang, "Mechanism studies on hepatotoxicity of rats induced by fructus toosendan," China Journal of Chinese Materia Medica, vol. 33, no. 16, pp. 2045-2047, 2008.

[125] T. Li, Z.-Z. Jiang, T. Wang et al., "Hepatotoxicity and its mechanism of saikosaponind on the human liver L02 cells in vitro," Chinese Journal of Clinical Pharmacology and Therapeutics, vol. 12, no. 4, pp. 396-400, 2007.
[126] D.-N. Qiu, Gene Regulations of Bile Acids Classic Synthesis Pathway, Fudan University, Shanghai, China, 2008.

[127] A.-H. Wang, L. Liu, and K. Zhou, "The progress in the study of the relation between cholestasis and bile acid transporters," Medical Recapitulate, vol. 19, no. 1, pp. 16-18, 2013.

[128] Z. H. Wang and Y. B. Qian, "Expression and significance of bsep and mrp2 after hepatic ischemia-reperfusion: experiment with rats," Journal of Hepatobiliary Surgery, vol. 19, no. 2, pp. 136-140, 2011.

[129] M. Trauner, M. Arrese, H. Lee, J. L. Boyer, and S. J. Karpen, "Endotoxin downregulates rat hepatic Ntcp gene expression via decreased activity of critical transcription factors," Journal of Clinical Investigation, vol. 101, no. 10, pp. 20922100, 1998.

[130] R. Green, D. Beier, and J. Gollan, "Regulation of hepatocyte bile salt transporters by endotoxin and inflammatory cytokines in rodents," Gastroenterology, vol. 111, no. 1, pp. 193-198, 1996.

[131] Y. Lv, X.-H. Sun, Q.-T. Chen et al., "Effect on rat liver about the compatibility of Polygonum multiflorum and black bean," Lishizhen Medicine and Materia Medica Research, vol. 24, no. 3, pp. 538-540, 2013.

[132] C. Zhang, Z.-Y. Liu, H.-J. Yu, and D.-L. Liu, "Phenolic acids from seed coats of Glycinemax," Chinese Traditional and Herbal Drugs, vol. 44, no. 24, pp. 3440-3443, 2013.

[133] Y.-L. Wang, H.-W. Ren, Z.-Z. Li, Y.-G. Wang, J.-L. Jiang, and X.-L. Dong, "Study on antioxidative activity evaluation medicinal black soybean pigments by scavenging DPPH," Science and Technology of Food Industry, vol. 30, no. 8, pp. 102-105, 2009.

[134] A.-J. Shen and W.-R. Cai, "The research progress on antiinflammatory effects and the therapy to acute lung injury of emodin," Journal of Zhejiang Chinese Medical University, vol. 37, no. 10, pp. 1261-1264, 2013.

[135] F.-X. Zhou, Intervention Effects of Emodin on Rats with Nonalcoholic Fatty Liver Disease Induced by Fat-Rich Diet and its Mechanisms, Central South University, Hunan, China, 2011.

[136] T.-X. Yao, The Effect of Emodin on Non-alcoholic Fatty Liver Rat Liver Injury Induced by Endotoxemia, Central South University, Hunan, China, 2011.

[137] L. L. Zhang, X. J. Li, and J. T. Jia, "Effect of emodin on the oxidative stress in the liver of hepatic fibrosis rats," Journal of Changzhi Medical College, vol. 28, no. 2, pp. 81-84, 2014.

[138] Y. H. Wang, H. P. Zhao, J. B. Wang, Y. L. Zhao, and X. H. Xiao, "Study on dosage-toxicity/efficacy relationship of prepared rhubarb on basis of symptom-based prescription theory," China Journal of Chinese Materia Medica, vol. 39, no. 15, pp. 2918-2923, 2014.

[139] J. Y. Pang, Z. F. Bai, M. Niu et al., "The toxic and protective effects of Polygonum multiflorum on normal and liver injured rats based on the symptom-based prescription theory," Acta Pharmaceutica Sinica, vol. 50, no. 8, pp. 973-979, 2015. 